\title{
ON THE ENDOMORPHISM NEAR-RING OF A FREE GROUP
}

\author{
by R. WARWICK ZEAMER \\ (Received 19th September 1978)
}

\section{Introduction}

Suppose $F$ is an additively written free group of countably infinite rank with basis $T$ and let $E=\operatorname{End}(F)$. If we add endomorphisms pointwise on $T$ and multiply them by map composition, $E$ becomes a near-ring. In her paper "On Varieties of Groups and their Associated Near Rings" Hanna Neumann studied the sub-near-ring of $E$ consisting of the endomorphisms of $F$ of finite support, that is, those endomorphisms taking almost all of the elements of $T$ to zero. She called this near-ring $\Phi_{\omega}$. Now it happens that the ideals of $\Phi_{\omega}$ are in one to one correspondence with varieties of groups. Moreover this correspondence is a monoid isomorphism where the ideals of $\Phi_{\omega}$ are multiplied pointwise. The aim of Neumann's paper was to use this isomorphism to show that any variety can be written uniquely as a finite product of primes, and it was in this near-ring theoretic context that this problem was first raised. She succeeded in showing that the left cancellation law holds for varieties (namely, $U(V)=U^{\prime}(V)$ implies $\left.U=U^{\prime}\right)$ and that any variety can be written as a finite product of primes. The other cancellation law proved intractable. Later, unique prime factorization of varieties was proved by Neumann, Neumann and Neumann, in (7). A concise proof using these same wreath product techniques was also given in $H$. Neumann's book (6). These proofs, however, bear no relation to the original near-ring theoretic statement of the problem.

The present paper originated in an attempt to find a near-ring theoretic proof of unique prime factorization of varieties. In the course of this it was found that not only does the set $\underline{V}$, of varieties (or equivalently, fully invariant subgroups of $F$ ) possess a natural monoid structure, but this can be extended to an equally natural multiplication on $\underline{C}$, the set of characteristic subgroups of $F$. Moreover, all results that could be obtained near-ring theoretically about the arithmetic of $\underline{V}$ could also be obtained for $\underline{C}$. As a further indication that arithmetic in $\underline{C}$ is similar to arithmetic in $\underline{V}$, Lemma 23.21 of (6), which $H$. Neumann uses to prove unique prime factorization of varieties, can be restated verbatim for characteristic subgroups, and a proof of this would amount to a proof of unique prime factorization in $\underline{C}$.

The advantage of considering the full endomorphism near-ring $E$ instead of $\Phi_{\omega}$ for the study of subgroups of $F$, is that the ordered bases of infinite rank subgroups are elements of $E$. The set of ordered bases for elements of $C$, denoted $B C$, turns out to be a multiplicative subset of $E$ and contains a submonoid $B V$, consisting of the ordered bases of elements of $\underline{V}$. The problem of unique prime factorization in $\underline{C}$ amounts to the problem of unique prime factorization up to multiplication by units in 
$B C$. As a possible indication that unique prime factorization holds in $\underline{C}$ we can show that both cancellation laws hold in $B C$.

Arithmetically, $\underline{V}$ is a very special submonoid of $\underline{C}$. In fact,

$$
K, K^{\prime} \in \underline{C}, K\left(K^{\prime}\right) \in \underline{V} \text { implies that } K, K^{\prime} \in \underline{V} .
$$

This means that unique prime factorization in $C$ implies unique prime factorization in $\underline{V}$. The wreath product proof of unique prime factorization in $\underline{V}$ does not extend to a proof of that result for $\underline{C}$. We conjecture that unique prime factorization does hold in $\underline{C}$ but it is likely that much deeper methods than those developed in this paper will be necessary to prove this.

The rest of the paper is divided into two sections. In the first we give some purely near-ring theoretic results about $E$. We show, for instance, that the set of two sided ideals of $E$ is a monoid under pointwise multiplication and that the closed ideals of $E$ form a monoid isomorphic to $\underline{V}$. In the second section we investigate the arithmetic of $\underline{C}$.

\section{The Near-Ring End $(F)$}

For a set $A$, let $Z(A)$ denote the free group on $A$. Let $T=\left\{t_{i}\right\}_{i=1}^{\infty}$ be a countably infinite set and let $F=F_{\infty}=Z(T)$. Let $E$ be the near-ring with underlying set $\operatorname{End}(F)$ and with addition and multiplication defined as follows:

For $f, g \in \operatorname{End}(F),(f+g)(t)=f(t)+g(t)$ for all $t \in T$ and $f \circ g=f \circ g$, where maps are composed on the left.

Let $S=\left\{s_{i}\right\}_{i=1}^{\infty}$ be a countably infinite set disjoint from $T$. For $t_{i} \in T$, let $\bar{t}_{i}=s_{i}$ and $\bar{s}_{i}=t_{i}$. The elements of $E$ can be represented uniquely as infinite sums of the form: $\sum_{i=1}^{\infty} w_{i} s_{i}, w_{i} \in F$. Addition is given by $\left(\sum w_{i} s_{i}\right)+\left(\Sigma u_{i} s_{i}\right)=\Sigma\left(w_{i}+u_{i}\right) s_{i}$; multiplication by $\left(\Sigma w_{i} s_{i}\right) \circ\left(\Sigma u_{i}\left(t_{i}\right) s_{i}\right)=\Sigma u_{i}\left(w_{i_{i}}\right) s_{i}$. We will use this infinite sum notation throughout this paper, $\Sigma w_{i} s_{i}$ is understood to mean $\Sigma_{i=1}^{\infty} w_{i} s_{i}$.

If we let $F$ have the discrete topology and then let $F^{\omega}=E$ have the induced product topology, $P,(E, P)$ is a topological near-ring. Note that $x_{i} \rightarrow x$ in $P$ if and only if for $N \geqslant 1$ there exists an $M \geqslant 1$ such that $i \geqslant M$ implies $\left(x_{i}\right)_{j}=(x)_{j}$ for $1 \leqslant j \leqslant N$. It is easy to see from this that $a_{i} \rightarrow a, b_{i} \rightarrow b$ in $P$ implies that $a_{i}+b_{i} \rightarrow a+b, a_{i} \circ b_{i} \rightarrow a \circ b$ in $P$. Since $P$ has a countable basis addition and multiplication are continuous in $P$. From now on, by a convergent sequence in $E$ we mean a sequence convergent with respect to $P$.

Using the above topology we can define in $E$ both infinite sums and infinite products though in both cases we must specify the direction in which the sum or product is taken.

For $f \in E$, let $(f)_{i}=f\left(t_{i}\right)$. Given $\left\{f_{i}\right\}_{i=1}^{\infty} \subseteq E, \Sigma_{i=1}^{\rightarrow \infty} f_{i}$ is the limit of $\left\{f_{1}+\cdots+f_{n}\right\}_{n \geqslant 1}$; $\sum_{i=1}^{+\infty} f_{i}$ is the limit of $\left\{f_{n}+\cdots+f_{1}\right\}_{n \geqslant 1}$. Whenever we write $\sum_{i=1}^{\infty} f_{i}$ we mean $\sum_{i=1}^{\infty} f_{i}$. Also define $\prod_{i=1}^{\rightarrow \infty} f_{i}=\lim \left\{f_{1} f_{2} \ldots f_{n}\right\}_{n \geqslant 1} \prod_{i=1}^{\rightarrow \infty} f_{i}=\lim \left\{f_{n} \ldots f_{2} f_{1}\right\}_{n \geqslant 1}$. Let $e=$ id $_{F}$ be $E$ 's multiplicative identity. 
Proposition 1. Let $\left\{f_{i}\right\}_{i=1}^{\infty} \subseteq E$.

(a) $\sum_{i=1}^{\infty} f_{i}$ converges if and only if for $N \geqslant 0$ there is an $M \geqslant 0$ such that for $i \leqslant M$, $\left(f_{i}\right)_{N}=0$. Moreover, $\sum_{i=1}^{\rightarrow \infty} f_{i}$ converges if and only if $\sum_{i=1}^{\rightarrow \infty}-f_{i}$ converges, if and only if $\sum_{i=1}^{\rightarrow \infty} f_{i}$ converges.

(b) $\prod_{i=1}^{\infty} f_{i}$ converges if and only if for $N \geqslant 1$ there is an $M \geqslant 1$ such that $\left(f_{M} \ldots f_{1}\right)_{N}$ is a fixed point of $f_{i}$ for $i>M$.

(c) $f_{i} \rightarrow e$ implies $\prod_{i=1}^{\rightarrow \infty} f_{i}$ converges.

Proof. (a) $\sum_{i=1}^{\rightarrow \infty} f_{i}$ converges if and only if $\left\{\sum_{i=1}^{n} f_{i}\right\}_{n=1}$ converges, if and only if for all $N \geqslant 1$ there exists an $M \geqslant 1$ such that $j, i \geqslant M \Rightarrow \sum_{k=1}\left(f_{k}\right)_{N}=\sum_{k=1}^{i}\left(f_{k}\right)_{N}$. For all $N \geqslant 1$, there exists an $M \geqslant 1$ such that $i \leqslant M \Rightarrow\left(f_{i}\right)_{N}=0$. Therefore $\sum_{i=1} f_{i}$ converges $\Leftrightarrow \sum_{i=1}^{\rightarrow \infty}-f_{i}$ converges $\Leftrightarrow\left\{\sum_{i=1}^{m}-f_{i}\right\}_{n \geqslant 1}$ converges $\Leftrightarrow\left\{\sum_{i=n} f_{i}\right\}_{n \geqslant 1}$ converges $\Leftrightarrow \sum_{i=1}^{\rightarrow \infty} f_{i}$ converges.

(b) $\prod_{i=1}^{-\infty} f_{i}$ converges $\Leftrightarrow\left\{f_{n} \ldots f_{1}\right\}_{n \geqslant 1}$ converges $\Leftrightarrow$ for all $N \geqslant 1$ there exists an $M \geqslant 1$ such that $i, j \geqslant M$ implies $\left(f_{i} \ldots f_{1}\right)_{N}=\left(f_{i} \ldots f_{1}\right)_{N} \Leftrightarrow$ for all $N \geqslant 1$ there exists $M \geqslant 1$ such that $i \geqslant M \Rightarrow\left(f_{i} \ldots f_{1}\right)_{N}=\left(f_{M} \ldots f_{1}\right)_{N}$, i.e. $f_{i}\left(f_{M} \ldots f_{1}\right)_{N}=\left(f_{M} \ldots f_{1}\right)_{N}$ for all $i \geqslant M$.

(c) Let $f_{i} \rightarrow e$. Then for $N \geqslant 1$ there exists $M \geqslant 1$ such that $i \geqslant M$ implies $\left(f_{i}\right)_{N}=t_{N}$. Therefore $i \geqslant M$ implies $\left(f_{1} \ldots f_{i}\right)_{N}=\left(f_{1} \ldots f_{M}\right)_{N}$. Hence for $N \geqslant 1$ there exists an $M \geqslant 1$ such that $i, j \geqslant M \Rightarrow\left(f_{1} \ldots f_{i}\right)_{N}=\left(f_{1} \ldots f_{j}\right)_{N}$. Hence $\prod_{i=1}^{\rightarrow \infty} f_{i}$ converges.

We now use infinite products to express any automorphism of $Z(T)$ in terms of elementary Nielsen transformations. We take all facts concerning Nielsen reduced subsets of $F$ from Section 3.2 of (4). Following the definition given there we define an elementary Nielsen transformation in our notation as follows:

Definition 1. An elementary Nielsen transformation is an element of $E$ of one of the following forms:

(1) $\Sigma_{k \neq i} t_{k} s_{k}+\left(t_{i}+t_{i}\right) s_{i}$ where $i \neq j$.

(2) $\Sigma_{k \neq i, j} t_{k} s_{k}+t_{i} s_{j}+t_{j} s_{i}$ where $i \neq j$.

(3) $\Sigma_{k \neq i} t_{k} s_{k}+-t_{i} s_{i}$.

Let Aut denote the group of automorphisms of $F$. Let $N=\{1,2,3, \ldots\}$. For any $\tau \in$ Aut, $\tau=\sum_{i \in N-A} t_{i} s_{i}+\sum_{i \in A} \tau_{i} s_{i}$ where $\tau_{i} \neq t_{i}$ for all $i \in A$. We write $\tau$ as $\tau=\sum_{i \in A} \tau_{i} s_{i}$ with the understanding that for $i \notin A(\tau)_{i}=t_{i}$. Now for $i \neq j, \quad\left(t_{i}+\epsilon t_{j}\right) s_{i}=$ $\left(\epsilon t_{j} s_{j}\right)\left(\left(t_{i}+t_{j}\right) s_{i}\right)\left(\epsilon t_{j} s_{j}\right)$. Similarly $\left(\epsilon t_{j}+t_{i}\right) s_{i}$ can be written as a product of elementary Nielsen transformations. Thus any automorphism of $F$ fixing all but finitely many elements of $T$ can be written as a finite product of elementary Nielsen transformations. (See Section 3.2 of (4).)

Proposition 2. (a) With the relative topology from E, Aut is a topological group.

(b) $\prod_{i=1}^{\vec{\infty}} \tau_{i}=\tau, \prod_{i=1}^{\rightarrow \infty} \sigma_{i}=\sigma$, where $\sigma_{i}, \tau_{i}, \sigma, \tau \in$ Aut, implies

$$
\begin{aligned}
& \tau^{-1}=\prod_{i=1}^{\leftarrow \infty} \tau_{i}^{-1}, \\
& \sigma^{-1}=\prod_{i=1}^{\infty} \sigma_{i}^{-1} .
\end{aligned}
$$


(c) Every $\tau \in$ Aut can be represented in the form,

$$
\tau=\prod_{i=1}^{\infty} \tau_{i}=\prod_{i=1}^{\leftarrow \infty} \tau_{i}^{\prime}
$$

where $\tau_{i}, \tau_{i}^{\prime}$ are elementary Nielsen transformations.

Proof. (a) As $E$ is a topological near-ring, we need only show that $\tau \mapsto \tau^{-1}$ is a continuous map Aut $\rightarrow$ Aut. For this it suffices to show that $\tau_{i} \rightarrow \tau$ implies $\tau_{i}^{-1} \rightarrow \tau^{-1}$.

Now if $x_{i} \in$ Aut and $x_{i} \rightarrow e$, then for all $N \geqslant 1$ there exists an $M \geqslant 1$ such that $i \geqslant M$ implies that for $1 \leqslant j \leqslant N\left(x_{i}\right)_{j}=t_{j}$ and so $\left(x_{i}^{-1}\right) j=t_{j}$. Therefore $x_{i} \rightarrow e$ if and only if $x_{i}^{-1} \rightarrow e$. Since multiplication is continuous in $E, \tau_{i} \rightarrow \tau \Rightarrow \tau^{-1} \tau_{i} \rightarrow e \Rightarrow \tau_{i}^{-1} \tau \rightarrow e \Rightarrow \tau_{i}^{-1} \rightarrow$ $\tau^{-1}$.

(b) Since $\prod_{i=1}^{\rightarrow \infty} \tau_{i}=\tau$ where $\tau_{i}, \tau \in$ Aut, $\left\{\prod_{i=1}^{n} \tau_{i}\right\}_{n \geqslant 1} \rightarrow \tau$ and so by part (a) $\left\{\Pi_{i=n}^{1} \tau_{i}^{-1}\right\}_{n \geqslant 1} \rightarrow \tau^{-1}$. Thus $\Pi_{i=1}^{\leftarrow \infty} \tau_{i}^{-1}=\tau^{-1}$. Similarly, $\prod_{i=1}^{\leftarrow \infty} \sigma_{i}=\sigma$ implies that $\sigma^{-1}=$ $\prod_{i=1}^{\rightarrow \infty} \sigma_{i}^{-1}$.

(c) Given $\tau \in$ Aut, define $\sigma_{n} \in$ Aut by induction as follows: Let $\sigma_{1}=e$. Suppose $\sigma_{i}$ has been defined for $i \leqslant n, n \geqslant 1$. Let $p_{n}=\tau \sigma_{1} \ldots \sigma_{n}$ and let $\sigma_{n+1} \in$ Aut be such that $\sigma_{n+1}\left(t_{i}\right)=t_{i}$ for all $i>n+1$ and $\left(\left(p_{n} \sigma_{n+1}\right)_{1}, \ldots,\left(p_{n} \sigma_{n+1}\right)_{n+1}\right)$ is the result of Nielsen reducing $\left(\left(p_{n}\right)_{1}, \ldots,\left(p_{n}\right)_{n+1}\right)$ and putting those $t_{i}$ that occur in this result first, arranging them in the order of their indices. One can verify by induction on $n$ that

$$
\operatorname{gp}\left(\left(p_{n}\right)_{1}, \ldots,\left(p_{n}\right)_{n}\right)=\operatorname{gp}\left((\tau)_{1}, \ldots,(\tau)_{n}\right) .
$$

Thus for $N \geqslant 1$ there exists an $M \geqslant 1$ such that $M^{\prime} \geqslant M$ implies $t_{1}, \ldots, t_{N} \in \operatorname{gp}\left(\tau_{i}\right)_{i=1}^{M^{\prime}}$ and so $\left(\left(p_{M^{\prime}}\right)_{1}, \ldots,\left(p_{M^{\prime}}\right)_{M^{\prime}}\right)=\left(t_{1}, \ldots, t_{N^{\prime}}\left(p_{M^{\prime}}\right)_{N+1}, \ldots,\left(p_{M^{\prime}}\right)_{M^{\prime}}\right)$. This follows from the fact that if $t \in T$ is an element of a subgroup $H$ of $F$ and $B$ is a Nielsen reduced basis for $H$, then $\pm t \in B$. Thus $\left\{p_{n}\right\}_{n \geqslant 1} \rightarrow e$, and so $\Pi_{i=1}^{\rightarrow \infty} \sigma_{i}=\tau^{-1} \tau=\Pi_{i=1}^{\leftarrow \infty} \sigma_{i}^{-1}$. Each $\sigma_{i}^{-1}$ is a finite product of elementary Nielsen transformations we have half of our result. The other half is obtained by applying our representation for $\tau$ to $\tau^{-1}$ and then using part (b).

We now apply the theory of Nielsen transformations to characterise the idempotents of Hanna Neumann's near-ring $\Phi_{\omega}=\left\{f \in E \mid(f)_{i}=0\right.$ for almost all $\left.i\right\}$. For $x \in E$ we write $(x)_{t}=x_{t}=x(t)$ and $x_{i}=(x)_{i}=(x)_{i}$ as long as this causes no confusion.

Proposition 3. For $a \in \Phi_{\omega}, a^{2}=a$ if and only if $a=\tau f \tau^{-1}$ where $\tau \in$ Aut and $f \in \Phi_{\omega}$ is of the form $f=\Sigma_{t \in A}\left(t+K_{t}\right) \bar{t}, A$ a finite subset of $T, K_{t} \in \operatorname{ngp}(T-A)$ for $t \in A$.

Proof. $a=\Sigma_{t \in B} a_{t} \bar{t}$ where $B=\left\{t \in T \mid a_{t} \neq 0\right\}$ is finite. Now there exists an $x \in$ Aut such that $x_{t}=t$ for $t \notin B$ and $\left\{(a x)_{t} \neq 0 \mid t \in B\right\}$ is Nielsen reduced. Let $A=$ $\left\{t \in T \mid(a x)_{t} \neq 0\right\} \subseteq B$. Then $f=x^{-1} a x=\Sigma_{t \in A} x^{-1}\left((a x)_{t}\right) \bar{t}$ is idempotent with kernel $\operatorname{ngp}(T-A)$ and $\left.\left.\left\{f_{t}\right\}_{t \in A}=\right\}\left(x^{-1} a x\right)_{t}\right\}_{t \in A}$ is free.

$f^{2}=f \Rightarrow f(f-e)=0$. For $t \in A, f_{t}-t$ is in $\operatorname{ngp}(T-A)$ so $f_{t}=t+K_{t}$ where $K_{t} \in$ $\operatorname{ngp}(T-A)$. Therefore $f=\Sigma_{t \in A}\left(t+K_{t}\right) \bar{t}$ and $a=x f x^{-1}$, completing the proof.

Problem. The above characterisation of the idempotents of $\Phi_{\omega}$ can be extended to the whole of $E$ if the following is true: 
$f \in E \Rightarrow$ there exists an $x \in$ Aut such that $\left\{(f x)_{t} \neq 0 \mid t \in T\right\}$ is free. This is equivalent to saying that if $f \in \operatorname{End}(F), \operatorname{Ker}(f)=\operatorname{ngp}\left(B^{\prime}\right)$ for some $B^{\prime} \subseteq B, B$ is a basis for $F$. As yet we have been unable to prove or disprove this.

Though $E$ is not d.g., it is topologically d.g., as noted by Tharmaratnam in (8). Thus every $f \in E$ can be written as an infinite convergent sum of distributive elements.

Consider $\left\{t_{i} s_{j}\right\}_{i, j \geqslant 1}$. These are clearly distributive elements. For $f \in E, f=$ $\sum_{i=1}^{\infty} w_{i}\left(t_{i_{j}}\right) s_{i}=w_{1}\left(t_{1 j} s_{1}\right)+w_{2}\left(t_{2 j} s_{2}\right)+\cdots+w_{i}\left(t_{i j} s_{i}\right)+\cdots$ which is clearly a convergent sum of distributive elements. This infinite sum representation gives multiplication in $E$ the same convenient structure it has in a d.g. near ring.

Proposition 4. The monoid of distributive elements of $E$ is $D=\left\{\sum_{i=1}^{\infty} d_{i} s_{i} \mid d_{i} \in T^{0}\right\}$, where $T^{0}=T \cup\{0\}$.

Proof. Let $D$ denote $E$ 's monoid of distributive elements. Clearly $x \in T^{0}$ implies $x s_{i} \in D$. Thus if $d=\sum_{i=1}^{\infty} d_{i} s_{i}, d_{i} \in T^{0}$ then if $a, b \in E$,

$$
(a+b) d=\sum_{i=1}^{\infty}(a+b)\left(d_{i} s_{i}\right)=\sum_{i=1}^{\infty}\left(a\left(d_{i} s_{i}\right)+b\left(d_{i} s_{i}\right)\right)=a d+b d,
$$

since multiplication in $E$ is continuous.

Now suppose $d \in D$. Since $t_{i} s_{i} \in D$ we have $d_{i} s_{i} \in D$. Let $d_{i}=w\left(t_{i 1}, \ldots, t_{\text {in }}\right)$ in $F$ and let $x=\sum_{j=1}^{n} t_{i j} s_{i j}$. For $k \geqslant 1,(k x)\left(d_{i} s_{i}\right)=k\left(x\left(d_{i} s_{i}\right)\right)$ and so $w\left(k t_{i 1}, \ldots, k t_{i n}\right)=k w$. Thus $w=M t$ for some $M \in Z$ and $t \in T$. Mts $s_{i} \in D$ implies that $\left(t_{i} \bar{t}+t_{2} \bar{t}\right) M t s_{i}=$ $\left(M t_{1}+M t_{2}\right) s_{i}=M\left(t_{1}+t_{2}\right) s_{i}$. Hence $M$ is 1 or 0 and $d_{i} \in T^{0}$, completing the proof.

We will now consider the ideal theory of $E$. First we need some notation. If $x \in E$, denote $\left\{x_{i} \mid i \geqslant 1\right\}$ by $\operatorname{cmp}(x)$. Let $\mathrm{gp}(x)=\operatorname{gp}(\operatorname{cmp}(x))$ and $\operatorname{ngp}(x)=\operatorname{ngp}(\operatorname{cmp}(x))$. If $A \subseteq E$, let $\mathrm{gp}(A)=\mathrm{gp}\left(\cup_{a \in A} \operatorname{cmp}(a)\right)$ and $\mathrm{ngp}(A)=\operatorname{ngp}\left(\cup_{a \in A} \overline{\mathrm{cmp}}(a)\right)$.

Note that the underlying group of the near-ring $E$ is $F^{\omega}$ so we will often refer to subsets of $E$ of the form $H^{(\omega)}$ where $H$ is a subset of $F$ and $H^{(\omega)}=H^{\omega} \cap \Phi_{\omega}$.

Definition 2. For $A \subseteq E, A$ is left closed if $E \cdot A \subseteq A$. $A$ is right closed if $A \cdot E \subseteq A . A$ is two-sided if $A$ is right and left closed.

Remark 1. For $x \in E, x \cdot E=g p(x)^{\omega}$.

Remark 2. For a subgroup $H$ of $F, H^{\omega} \subseteq E$ is a right closed subgroup of $E$.

Proposition 5. The following are equivalent:

(1) $A \subseteq E$ is an ideal;

(2) $A \subseteq E$ is a two-sided normal subgroup of $E$;

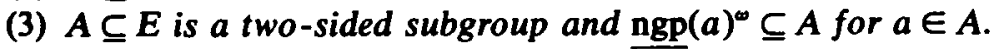

Proof. (1) $\Rightarrow$ (2): If $A \subseteq E$ an ideal then $A<E, E A=A$ and for $x, y \in E, a \in A$, $(x+a) y-x y \in A$. Letting $x=0$, we have that $A$ is right closed.

(2) $\Rightarrow$ (3): Since $|S|=\omega$ we may write $S$ as a disjoint union, $S=\cup_{f \in F}\left\{s_{i f}\right\}_{i=1}^{\infty}$. Let $x=\Sigma_{f \in F} \Sigma_{i=1}^{\infty} f s_{i_{f}} \in E, y=\Sigma_{f \in F} \sum_{i=1}^{\infty} t_{i} s_{i_{f}} \in E$. Take $a \in A . z=x+a y-x \in A$ so for 
$f \in F, i \geqslant 1 z_{i,}=x_{i f}+(a y)_{i,}-x_{i f}=f+a_{i}-f$. By Remark $1, z E=\operatorname{gp}(z)^{\omega}=\operatorname{ngp}(a)^{\omega} \subseteq A$.

(3) $\Rightarrow(1)$ : We need only show that $x, y \in E, a \in A$ implies that $(x+a) y-x y \in A$. But $(x+a) y-x y=d+x y-x y$ where $d \in \operatorname{ngp}(a)^{\omega} \subseteq A$. This completes the proof.

We now give explicit formulas for the two-sided subgroup, ideal, and right closed subgroup generated by a subset of $E$.

Proposition 6. For any $A \subseteq E$,

(1) The two-sided subgroup generated by $A$ is

$$
R L(A)=\cup\left\{\underline{g p}(B)^{\omega} \mid B \text { a finite subset of } E A\right\} .
$$

(2) The ideal generated by $A$ is

$$
\operatorname{Ideal}(A)=\cup\left\{\underline{\operatorname{ngp}}(B)^{\nu} \mid B \text { a finite subset of } E A\right\} .
$$

(3) The right closed subgroup generated by $A$ is

$$
R(A)=\cup\left\{\underline{g p}(B)^{\omega} \mid B \text { a finite subset of } A\right\} .
$$

Proof. (1): $x, y \in R L(A) \Rightarrow x \in \mathrm{gp}(B)^{\omega}, \quad y \in \mathrm{gp}(C)^{\omega}, B, C \subseteq E A$ finite $x+y \in$ $\operatorname{gp}(B \cup C)^{\omega}$ and so $x+y \in R L(A)$. Thus $R L(A)$ is a subgroup and similarly $\operatorname{Ideal}(A)$ and $R(A)$ is a subgroup. All three sets are right closed by Remark 2. Therefore $R(A)$ is a right closed subgroup.

For $x \in E$ and a finite $B \subseteq E A, x \operatorname{ngp}(B)^{\omega} \subseteq \operatorname{ngp}(x B)^{\omega}$ and $x+\operatorname{ngp}(B)^{\omega}-x$ is contained in $\operatorname{ngp}(B)^{\omega}$. Hence Ideal $(A)$ is an ideal of $E$. Similarly one can show that $R L(A)$ is a two-sided subgroup of $E$.

Now suppose $K \supseteq A$, where $K$ is a two-sided subgroup of $E$. Then $K \supseteq E A$. Suppose that $\left\{b_{1}, \ldots, b_{n}\right\}=B \subseteq E A$, finite. Write $S=\cup \cup_{i=1}^{m}\left\{s_{i j}\right\}_{j=1}^{\infty}$, a disjoint union of countably infinite sets. Let $f_{i}=\sum_{i=1}^{\infty} t_{i} s_{i j}$ for $1 \leqslant i \leqslant n$. Then $b=\sum_{i=1}^{n} b_{i} f_{i}$ is in $K$. Thus $b E=\mathrm{gp}(b)^{\omega}=\mathrm{gp}(B)^{\omega} \subseteq K$. This proves (1) and a similar argument proves (3).

To prove (2) suppose $K \supseteq A$ is an ideal. Take a finite $B \subseteq E A$. As in the proof of (1) we have $b \in K$ such that $\operatorname{gp}(b)=\mathrm{gp}(B)$. Hence $\operatorname{ngp}(b)=\operatorname{ngp}(B)$ and by Proposition $5, \underline{\operatorname{ng}}(B)^{\omega}=\operatorname{ngp}(b)^{\omega} \subseteq \bar{K}$. Therefore $K \supseteq \operatorname{Ideal}(A)$, completing the proof.

We now turn to the multiplicative structure of the set of two-sided subgroups of $E$ which we denote by $R L$.

Definition 3. For $A, B \in R L$, define $A \cdot B=A B=\left\{\sum_{i=1}^{n} a_{i} b_{i} \mid a_{i} \in A, b_{i} \in B\right\}$.

$A B$ is certainly a left closed subgroup of $E$ but we do not know a priori whether it is right closed since $E$ is not d.g.. The following theorem shows that it is. The proof is an adaption to $E$ of $H$. Neumann's proof that ideal multiplication is associative in $\Phi_{\omega}$.

Notation. For $w \in Z(X)$, let

$$
X(w)=\{x \in X \mid x \text { occurs in the reduced } X \text {-form of } w \text {. }\}
$$

For $f \in E$ let

$$
\operatorname{supp}(f)=\left\{t \in T \mid f_{t} \neq 0\right\}
$$


Theorem 1. $A, B \in R L \quad$ implies $A B=\left\{\sum_{i=1}^{m} a_{i} b_{i} \mid a_{i} \in A, b_{i} \in B\right\}=$ $\{a b \mid a \in A, b \in B\}$. Thus $R L$ forms a monoid under pointwise multiplication.

Proof. Take $A, B \in R L$. It suffices to show that for $a_{1}, a_{2} \in A, b_{1}, b_{2} \in B$,

$$
a_{1} b_{1}+a_{2} b_{2}=a b \text { for some } a \in A, b \in B \text {. }
$$

Since $F=Z(T)$ and $T$ is infinite we may take right distributive elements (Proposition 4) $f, f^{\prime}, g, g^{\prime}$ in $E$ such that $f f^{\prime}=g g^{\prime}=e$ and $T\left(f^{\prime}(F)\right) \cap \operatorname{supp}(g)=t\left(g^{\prime}(F)\right) \cap \operatorname{supp}(f)=$ 9 , and so $g f^{\prime}=g^{\prime} f=0$. Let $a=a_{1} f+a_{2} g, b=f^{\prime} b_{1}+g^{\prime} b_{2}$. Then $a \in A, b \in B$, and

$$
\begin{aligned}
a b & =\left(a_{1} f+a_{2} g\right) f^{\prime} b_{1}+\left(a_{1} f+a_{2} g\right) g^{\prime} b_{2} \\
& =\left(a_{1} f f^{\prime}+a_{2} g f^{\prime}\right) b_{1}+\left(a_{1} f g^{\prime}+a_{2} g g^{\prime}\right) b_{2} \\
& =\left(a_{1} e+a_{2} 0\right) b_{1}+\left(a_{1} 0+a_{2} e\right) b_{2}=a_{1} b_{1}+a_{2} b_{2} .
\end{aligned}
$$

This monoid $R L$ is also a lattice with respect to containment and can be written as a disjoint union of sublattices in the following way: Let $\underline{V}$ denote the set of fully invariant subgroups of $F$. Then

$$
R L=\cup_{V \in Y} R L_{V} \text {, where } R L_{V}=\left\{A \in R L \mid V^{(\omega)} \subseteq A \subseteq V^{\omega}\right\} .
$$

To see this suppose $A$ is right closed, $A \subseteq E$. For $i \geqslant 1$ define $p_{i}: E \rightarrow F$, an additive homomorphism by $p_{i}(f)=f_{i}$ for $f \in E . \quad A \cdot\left(t_{i} s_{j}\right)=p_{i}(A) s_{j} \subseteq A$ since $A$ is right closed. Hence $p_{i}\left(p_{i}(A) s_{j}\right) \subseteq p_{j}(A)$, and so $p_{i}(A) \subseteq p_{j}(A)$. Thus for all $i, j, p_{i}(A)=$ $p_{j}(A)=H$, a subgroup of $F$. Clearly $A \subseteq H^{\omega}$ and since $A \supseteq H s_{i}$ for all $i, A \supseteq H^{(\omega)}$. If $A$ is left closed $H \in \underline{V}$.

Theorem 2. (1) $R L_{V} \cdot R L_{U} \subseteq R L_{U(V)}$.

(2) The minimal and maximal ideals of $R L_{V}$ are $I_{V}$ and $V^{\omega}$ respectively, where $I_{V}=\bigcup\left\{\operatorname{ngp}(A)^{\omega} \mid A\right.$ a finite subset of $\left.V\right\}$.

(3) The minimal and maximal elements of $R L_{V}$ are $M V=\cup\left\{\operatorname{gp}(A)^{\omega} \| A\right.$ a finite subset of $V\}$ and $V^{\infty}$ respectively.

(4) For $U, V \in V, M_{V} \cdot M_{U}=M_{U(V)}$ and $V^{\omega} \cdot U^{\omega}=U(V)^{\omega}$. Thus $\left\{M_{V}\right\}_{V \in V}$ and $\left\{V^{\omega}\right\}_{V \in \underline{Y}}$ are submonoids of $R L$ anti-isomorphic to $\underline{V}$.

Proof. Take $A \in R L_{V}, B \in R L_{U}$. Then $V^{(\omega)} \cdot U^{(\omega)} \subseteq A B \subseteq V^{\omega} \cdot U^{\omega} \subseteq(U(V))^{\omega}$. H. Neumann proved in (5) that $V^{(\omega)} \cdot U^{(\omega)}=U(V)^{(\omega)}$. This proves (1).

(2): $V$ is clearly a two-sided normal subgroup of $E$ and hence the maximal ideal and the maximal element of $R L_{V}$. Following the proof of Proposition $6, I_{V}$ is easily checked to be an ideal of $E$. Suppose $K \in R L_{V}$ is an ideal. For $A=\left\{a_{1}, \ldots, a_{n}\right\} \subseteq V$,

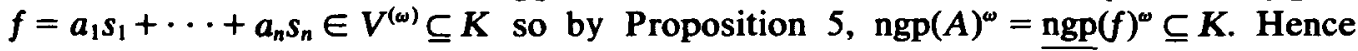
$I_{V} \subseteq K$. This proves (2).

(3): $M_{V}$ is easily checked to be in $R L_{V}$. Now if $K \supseteq V^{(\omega)}$ and $K \in R L_{V}$ then for $A=\left\{a_{1}, \ldots, a_{n}\right\} \subseteq V, f=a_{1} s_{1}+\cdots+a_{n} s_{n} \in V^{(\omega)} \subseteq K$. Hence $\operatorname{gp}(A)^{\omega}=\operatorname{gp}(f)^{\omega} \subseteq K$ and so $K \supseteq M_{V}$. This proves (3).

(4): By (1), $M_{V} \cdot M_{U} \supseteq M_{U(V)}$. Take $v \in M_{V}, u \in M_{U}$. Then $v \in \operatorname{gp}(A)^{\omega}, u \in \operatorname{gp}(B)^{\omega}$ where $A, B$ are finite, $A \subseteq V, B \subseteq U$. But $v u \in g \mathrm{gp}(v u)^{\infty} \subseteq \mathrm{gp}(v B)^{\omega}$. Therefore since $v B \subseteq U(V)$ is finite, $v u \in M_{U(V)}$. Hence $M_{V} \cdot M_{U} \subseteq M_{U(V)}$. 
It remains to show $U(V)^{\omega}=V^{\omega} \cdot U^{\omega}$ where $U, V \subseteq F$ are verbal. Now consider $f=\sum_{i=1}^{\infty} u_{i}\left(v_{i j}\right)_{j=1}^{n_{i}} s_{i} \in U(V)^{\omega}$. T $T=\cup_{i=1}^{\infty} T_{i}$, a disjoint union where each $T_{i}\left\{t_{i j}\right\}_{j=1}^{n_{i}} . f=$ $\left(\sum_{i=1}^{\infty} \sum_{j=1}^{n_{i}} v_{i j} \bar{t}_{i j}\right)\left(\sum_{i=1}^{\infty} u_{i}\left(t_{i j}\right) s_{i}\right) \in V^{\omega} \cdot U^{\omega}$. Thus $U(V)^{\omega} \subseteq V^{\omega} \cdot U^{\omega}$. On the other hand if $v=\sum_{i=1}^{\infty} v_{i} s_{i}$ and $u=\sum_{i=1}^{\infty} u_{i}\left(t_{i j}\right)_{j=1}^{n_{1}} s_{i}$, then $v \cdot u=\sum_{i=1}^{\infty} u_{i}\left(v_{i j}\right)_{j=1}^{n_{i}} s_{i} \in U(V)^{\infty}$. Hence $U(V)^{\omega}=V^{\omega} \cdot U^{\omega}$, proving (4).

Corollary 1. The closed ideals of E form a monoid anti-isomorphic to the monoid of varieties. The anti-isomorphism is: $V \exists V \mapsto V^{\omega}$.

Proof. In light of Theorem 2, we need only show that $A$ is a closed ideal of $E$ if and only if $A$ has form $V^{\omega}$ for some $V \in \underline{V}$. First $V^{\omega}$ is an ideal and it is clearly closed. If $A$ is a closed ideal then $A \in R L_{V}$ for some $V \in \underline{V}$ and so $V^{(\omega)} \subseteq A$. Now if $v \in V^{\omega}$, then for all $n \geqslant 1, \sum_{i=1}^{n} v_{i} s_{i}=p_{n} \in A$. Since $p_{n} \rightarrow v$ and $A$ is closed, $v \in A$. Therefore $A=V^{\omega}$.

\section{The Monoid of Characteristic Subgroups of $F$}

In this section we use $E$ to study the multiplicative structure of the set of subgroups of $F$.

Definition 1. For $M \subseteq E$ a semigroup, call $x \in E$ an $M$-element if $g \mathrm{p}(x)$ is $M$ invariant, that is, $M \cdot g p(x)$. In particular, if $x$ is an $E$-element we say $x$ is verbal. If $x$ is an Aut-element, we say $x$ is characteristic.

Definition 2. Suppose $K \subseteq F$ is infinite rank. We say $x \in E$ is basic for $K$ if $\operatorname{cmp}(x)$ is a basis for $K$. By convention, $0 \in E$ is basic for $\{0\}$. We call $x \in E$ free if $\operatorname{cmp}(x)$ is free.

Remark 1. If $M \subseteq E$ is a multiplicative semigroup then $x$ is an $M$-element if and only if $m \in M \Rightarrow m x=x m^{\prime}$ for some $m^{\prime} \in E$. free\}.

Remark 2. $\{x \mid x$ is basic for some $K \subseteq F, \operatorname{rank}(K)=\omega\}=\operatorname{Mon}(F, F)=\{x \in E \mid x$ is

Remark 3. The free elements of $E$ form a multiplicative monoid.

Now suppose that $K, U \subseteq F$ are subgroups where $K$ is of infinite rank and $U \neq 0$ is characteristic. Then we may take $k, u \in E$ such that $k$ is basic for $K, u$ is basic for $U$. Define the product of subgroups $K \cdot U=U(K)=\mathrm{gp}(k u)$. To show this product is well defined we must show it is independent of our choice of $k$ and $u$. So take $k^{\prime}$ and $u^{\prime}$ basic for $K$ and $U$ respectively. Then there are $x, y \in$ Aut such that $k^{\prime}=k x$ and $u^{\prime}=u y . k^{\prime} u^{\prime}=k x u y=k u x^{\prime} y$ and so $\underline{\operatorname{gp}}\left(k^{\prime} u^{\prime}\right) \subseteq \operatorname{gp}(k u)$. By symmetry we have equality and hence the product of subgroups, $\bar{U}(K)$, is well defined. Note that $k$ basic for $K, u$ basic for $U$ implies that $k u$ is basic for $U(K)$. Note also that according to our definitions, $O(K)=U(0)=0$. 
It happens that the above definition of $U(K)$ for $U$ characteristic extends the usual definition of $U(K)$ for $U$ verbal. To see this suppose $U$ is verbal and take $u$ basic for $U, k$ basic for $K . g p(k u)=k U \subseteq\left\{u\left(a_{1}, \ldots, a_{n}\right) \mid u \in U, a_{i} \in K\right\}$. But if $x=$ $u\left(a_{1}, \ldots, a_{n}\right)$ where $u \in U$ and $a_{i}=a_{i}\left(k_{i j}\right)_{j=1}^{n_{i}}, k_{i j} \in \operatorname{cmp}(k)$, then $U$ verbal implies $x \in \operatorname{gp}(k u)$. Therefore $\operatorname{gp}(k u)=\left\{u\left(a_{1}, \ldots, a_{n}\right) \mid u \in U, a_{i} \in K\right\}$, which is the usual definition of $U(K)$.

Proposition 1. The set $\underline{C}$ of characteristic subgroups of $F$ has a natural monoid structure extending the monoid structure on $\underline{V}$.

Proof. $\underline{C}$ will be multiplicatively closed if for $k$ basic for $K \in \underline{C} . x \in$ Aut implies that $x^{\prime}$ in $x k=k x^{\prime}$ is an element of Aut. But this must be so since $k$ and $x k$ are basic for $K$. Thus $\underline{C}$ is multiplicatively closed. Clearly it has identity $F$. It remains to show that the multiplication we have defined is associative: Take $K, H, L \in C$ with $k, h, l$ basic for $K, H, L$ respectively. $(K H) L=\underline{\mathrm{gp}}((k h) l)=\underline{\mathrm{gp}}(k(h l))=K(H L)$ since $h l$ is basic for $H L$.

We now introduce two multiplicative submonoids of $(E, \cdot)$ which will play a central role throughout the rest of this paper.

Let $B C=\{x \in E \mid x$ is basic for some $K \in C\}$, and let $B V=\{x \in E \mid x$ is basic for some $V \in \underline{V}\}$.

Proposition 2. $B V \subseteq B C$ are multiplicative submonoids of $E$ such that

(1) $B V=\left\{x \in E \mid\right.$ For $y \in E, y x=x y^{\prime}$ for some unique $\left.y^{\prime} \in E\right\}$.

(2) $B C=\left\{x \in E \mid\right.$ For $y \in$ Aut, $y x=x y^{\prime}$ for some $y^{\prime}$ unique in $\left.E\right\}$.

(3) $\mathrm{gp}: B C \rightarrow \underline{C}$ and $\mathrm{gp}: B V \rightarrow \underline{V}$ are monoid epimorphisms. For $a, b \in B C \mathrm{gp}(a)=$ $\underline{\mathrm{gp}}(\mathrm{b})$ if and only if $a=\overline{b x}$ for some $x \in$ Aut.

Proof. $a, b \in B V, f \in E$ implies $f a b=a f^{\prime} b=a b f^{\prime \prime}$ for some $f^{\prime}, f^{\prime \prime}$ in $E$ since $a, b$ are both $E$-elements. Hence $a b \in B V$ since $a, b$ are free. Now suppose $a, b \in B C$. $x \in$ Aut $\Rightarrow x a b=a x^{\prime} b=a b x^{\prime \prime}$ for some $x^{\prime}, x^{\prime \prime} \in$ Aut by the proof of Proposition 1. $a b$ is therefore characteristic. It is free since $a$ and $b$ are free. Thus $a b \in B C$.

Since for $x$ free $x z=x y$ implies $z=y$ and any $x$ basic for $K \in \underline{C}$ is an Autelement, $\subseteq$ holds in (2). Now take $x$ in the right hand side of (2). $g p(x)$ is characteristic and it remains to show that $x$ is free. If not, there exists a $k, 0 \neq k \in E$, such that $x k=0$. But then $e \cdot x=x \cdot e=x \cdot(e+k)$, a contradiction of the uniqueness of the $e^{\prime}$ such that $e \cdot x=x \cdot e^{\prime}$. Thus $x$ is free and hence in $B C$. (1) is proved similarly.

(3): Since gp: $B C \rightarrow \underline{C}$ is a monoid homomorphism by the definition of multiplication in $\underline{C}$ and is onto since every $0 \neq K \in \underline{C}$ has a countably infinite basis and 0 is basic for $0 \in \underline{C}$, the first statement of (3) is proved.

Suppose $a, b \in B C . \underline{\mathrm{gp}}(a)=\mathrm{gp}(b) \Rightarrow \mathrm{cmp}(a), \operatorname{cmp}(b)$ are bases for the same subgroup of $F \Rightarrow$ there exists $x \in \overline{\text { Aut }}$ such that $a=b x$.

Proposition 2 allows us to define the following multiplicative homomorphisms.

Definition 3. For $a \in B V$ define $Y_{a}: E \rightarrow E$ such that for $x \in E, x a=a(x) Y_{a}$. For 
$a \in B C$ define $C_{a}:$ Aut $\rightarrow$ Aut such that $x \in$ Aut implies that $x a=a(x) C_{a}$. Both maps are well defined by Proposition 2.

Proposition 3. (1) For $a \in B V, Y_{a} \mid$ Aut $=C_{a}$.

(2) For $a, b \in B V, Y_{a b}=Y_{a} \circ Y_{b}$; for $a, b \in B C, C_{a b}=C_{a} \circ C_{b}$.

(3) For $a \in$ Aut, $(x) Y_{a}=a^{-1} x a$ for all $x \in E$.

(4) For $a \in B V, Y_{a}: E \rightarrow E$ is a multiplicative homomorphism. For $a \in B C$, $C_{a}:$ Aut $\rightarrow$ Aut is a group homomorphism.

Proof. $B V \subseteq B C$ implies (1) is obvious from Definition 3.

(2): For $a, b \in B V, x \in E, a b(x) Y_{a b}=x a b=a(x) Y_{a} b=a b\left((x) Y_{a}\right) Y_{b}$. $a b$ free gives $Y_{a b}=Y_{a} \circ Y_{b}$. Similarly $C_{a b}=C_{a} \circ C_{b}$.

(3): $a \in$ Aut $\Rightarrow$ For $x \in E, x a=a a^{-1} x a=a(x) Y_{a}$ and so $a^{-1} x a=(x) Y_{a}$.

(4): $a \in B V$ implies that for $x, y \in E, x y a=a(x y) Y_{a}$. Also $x y a=x a(y) Y_{a}=$ $a(x) Y_{a}(y) Y_{a}$. Similarly $C_{a}$ is a group homomorphism for all $\alpha \in B C$.

We have laid the basis for our discussion of $\underline{C}$.

\subsection{Cancellation in $\underline{\boldsymbol{C}}$}

In this section we show that one cancellation law in $\underline{C}$ is trivial and that the real problem in the arithmetic of $\underline{C}$, as in the arithmetic of $\underline{V}$, is the proof of the other one. We are able to prove a weak form of this other cancellation law.

Proposition 4. Left cancellation holds in $\underline{C}$, that is, $K, H^{\prime}, H \in B C, K H=K H^{\prime}$ implies $\boldsymbol{H}=\boldsymbol{H}^{\prime}$.

Proof. If $k, h, h^{\prime}$ basic for $K, H, H^{\prime}$ respectively, $K H=\mathrm{gp}(k h)=\mathrm{gp}\left(k h^{\prime}\right)=K H^{\prime}$ implies that $k h=k h^{\prime} x$ for some $x \in$ Aut. But $k$ free implies $h=h^{\prime} x$ and so $H=H^{\prime}$.

We aim to prove the following weak form of the right cancellation law in $C$ : If $U \neq 0$ is in $\underline{C}$ and $K, K^{\prime}$ are subgroups of $F$ of infinite rank then

$$
K U=K^{\prime} U, K^{\prime} \subseteq K \Rightarrow K=K^{\prime} .
$$

We prove this by combinatorial methods.

Notation 1. For $w \in F$ let $|w|$ be the length of its $T$-reduced form.

2. For $u, v \in F, u+v$ is a reduced sum if $|u+v|=|u|+|v|$.

3. Recall from Section 3.2 of (4) that if $A \subseteq F, A$ is Nielsen reduced if and only if for $a, b, c \in \pm A, b \neq-a,-c,|a+b| \geqslant|a|,|b|$ and $|a+b+c|>|a|+|c|-|b|$. Note that this last condition implies that if $0 \in A, A=\{0\}$.

4. Suppose $A$ is Nielsen reduced, $A \subseteq F$. Then any $a \in A$ has the unique representation, $a=a_{0}+c(a)+a_{1}$, a reduced sum where $c(a)$, the core of $a$, is the section of $a$ none of whose symbols is cancelled in any reduced $A$-sum, $\epsilon a^{\prime}+a+$ $\delta a^{\prime \prime}, a^{\prime}, a^{\prime \prime} \in A, \epsilon, \delta= \pm$. Note $c(a) \neq 0$ since $A$ is Nielsen reduced and $c(a)$ is the section of $a$ not cancelled in any reduced word, $w\left(a, a_{1}, \ldots, a_{n}\right), a_{i} \in A$. (See Chapter I of (3).)

5. Suppose $0 \neq x \in F$. Let $m(x)$, the middle of $x$, be the non-zero section of $x$ of 
minimal length such that $x=x_{0}+m(x)+x_{1}$, is a reduced sum where $\left|x_{0}\right|=\left|x_{1}\right|$. Note $|m(x)|$ is one if $|x|$ is odd and two if $|x|$ is even.

6. For $A \subseteq F$ define $|A|=\min \{|a| \mid a \in A\}$.

Lemma 1. If $A \subseteq F, a$ Nielsen reduced set, then $a \in A$ implies $m(a)$ and $c(a)$ must overlap.

Proof. If $c(a)$ and $m(a)$ do not overlap we have:

(1)

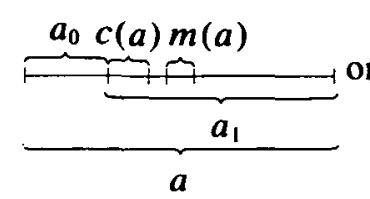

(2)

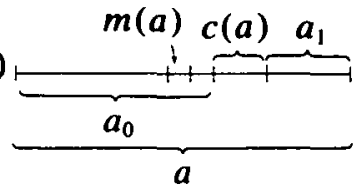

If (1) holds there exists $\epsilon a^{\prime} \in \pm A$ such that $\epsilon a^{\prime} \neq-a$ and in the sum, $a+\epsilon a^{\prime}, a_{1}$ is cancelled. But then $\left|a+\epsilon a^{\prime}\right|<\left|a^{\prime}\right|$, a contradiction of $A^{\prime}$ 's being Nielsen reduced. Case (2) is treated similarly.

The following lemma is the heart of our proof of the weak right cancellation law.

Lemma 2. If $U \neq 0$ in $\underline{C}$ and $a \in E$ is free then $a \cdot U \supseteq U$ implies $a \in$ Aut.

Proof. $\mathrm{gp}(a)$ is a free group of infinite rank and so has a Nielsen reduced basis $B$. Let $B=\left\{b_{i}\right\}_{i=1}^{\infty}$ and put $b=\sum_{i=1}^{\infty} b_{i} s_{i}$. Then there exists $x \in$ Aut such that $a x=b$. Thus $b U=a x U=a U \supseteq U$. For any $\epsilon t$ in $\pm T$ there exists a $u \in U$ of minimal length in $U$ such that $u=\epsilon t+u^{\prime}$, a reduced $T$-sum for some $u^{\prime} \in F$. Now there exists a $v\left(t_{i j}\right)_{j=1}^{n} \in$ $U$ such that $b v=v\left(b_{i j}\right)_{j=1}^{n}=u$. B Nielsen reduced $\Rightarrow|v|=|u|$ and each element of $B$ involved in $b v$ contributes exactly its core (which must have length one) to the reduced $T$-form of $u$. $v=\delta t_{i j}+v^{\prime}$ is a reduced $T$-sum where $v^{\prime} \in F$. Hence $u=$ $\delta b_{i j}+b v^{\prime}$ is a $B$-reduced sum. Thus $c\left(b_{i j}\right)=\epsilon t$ and by Lemma $1 \delta b_{i j}=\epsilon t+\eta t^{\prime}$, $\eta t^{\prime} \in \pm T^{0}$, where we have underlined the core of $b_{i j}$ in $\delta b_{i j}$. If $\eta t^{\prime} \neq 0$ then by repeating the same argument with $-\eta t^{\prime}$ in place of $\epsilon t$ we get a $\gamma b_{k} \in \pm B$ such that $\gamma b_{k}=-\eta t^{\prime}+\xi t^{\prime \prime}$. Since $-\eta t^{\prime}$ is the core of $\gamma b_{k}$ it can only cancel in an unreduced $B$-sum. Thus we have $\delta b_{i j}=-\gamma b_{k}=-\xi t^{\prime \prime}+\underline{\eta t^{\prime}}=\underline{\epsilon t}+\eta t^{\prime}$. But this puts the core of $b_{i j}$ in two mutually exclusive places. Thus $\eta t^{\prime}=0$ and for any $\epsilon t$ in $\pm T$ there is a $\delta b_{i j} \in \pm B$ such that $\delta b_{i j}=\epsilon t$. Thus $\underline{\mathrm{gp}}(a)=\underline{\mathrm{gp}}(b)=F$ and so $a \in$ Aut.

Theorem 3. If $U \neq 0$ is characteristic and $K, K^{\prime} \subseteq F$ are subgroups of infinite rank then $K^{\prime} \subseteq K, K^{\prime} U \supseteq K U$ implies $K=K^{\prime}$.

Proof. Let $u, k, k^{\prime}$ be basic for $U, K, K^{\prime}$ respectively. $k u$ and $k^{\prime} u$ are basic for $K U$ and $K^{\prime} U$ respectively. Thus since $K^{\prime} U \supseteq K U$, there exists an $x \in E$ such that $k u=k^{\prime} u x$. Since $K^{\prime} \subseteq K$ there exists $y \in E$ such that $k y=k^{\prime} . k^{\prime}$ free implies $y$ is free. Hence $k u=k^{\prime} u x=k y u x \Rightarrow u=y u x \Rightarrow U \subseteq y U$ so by Lemma $2 y \in$ Aut. So $K=K^{\prime}$, completing the proof. 


\subsection{On $Y_{a}$ and $C_{a}$}

In this section we will use the techniques of Section 2.1 to show that for $a \in B C$, $C_{a}:$ Aut $\rightarrow$ Aut is a group monomorphism which is epi if and only if $a \in$ Aut.

Lemma 3. Suppose $K$ is a subgroup of $F . K_{0}=\{k \in K|0 \neq| k \mid$ minimal $\}$. Then $K_{0}$ consists of primitive elements of $K$ and if $B$ is a Nielsen reduced basis for $K, k \in K_{0}$ is either in $\pm B$ or of the form $k=\epsilon b_{i}+\delta b_{2}$, where $b_{1} \neq b_{2}$ are in $B \subseteq K_{0}$.

Proof. This is simply Corollary 3.4 of (4).

Lemma 4. If $0 \neq H \subseteq K \subseteq F$, where $H$ is characteristic in $K$, then $|H|>|K|$.

Proof. $|H|=|K|$ implies there exists $k \in K_{0} \cap H$. By Lemma $3 k$ is primitive in $K$ and so since $H$ is characteristic in $K, H=K$. Thus $|H|>|K|$.

Lemma 5. A monomorphism $F \rightarrow F$ which fixes the elements of minimal length of a non-zero characteristic subgroup must be the identity.

Proof. Take $f \in E$ free such that $f a=a$ for all $a \in K_{0}$, where $0 \neq K$ is in $C$. Note $P K_{0}=K_{0}$ where $P=\left\{\Sigma \epsilon_{i} t_{f(i)} s_{i} \mid f\right.$ a bijection of $\left.N\right\}$. Fix $k \in K_{0}$ and let $A=T(k)=$ $\left\{t_{i j}\right\}_{j=1}^{n}$. There exists $x \in$ Aut such that $x_{r}=t_{r}$ for $r$ such that $t_{r} \notin T(k), T\left(x_{i j}\right) \subset A$ for $1 \leqslant j \leqslant n$, and $f x=y$ where $\left(y_{i 1}, \ldots, y_{i n}\right)$ is the Nielsen reduction of $\left(f_{i 1}, \ldots, f_{i n}\right)$. For all $p \in P$ such that $p k \in Z(A)$ we have $y x^{-1} p k=p k$. Note also that $T\left(x^{-1} p k\right) \subseteq A$. Since $x^{-1} p k \in K$ and $\left(y_{i j}\right)_{j=1}^{n}$ is Nielsen reduced $\left|x^{-1} p k\right|=|p k|=|k|$. Moreover $y x^{-1} p k=$ $p k$, and so each $y_{i j}$ contributes exactly its core (which must have length one) to the reduced $T$-form of $p k$. For any $\epsilon t_{i j} \in \pm A$ we may pick $p k=\epsilon t_{i j}+h \in Z(A)$ a reduced $T$-sum. Thus there is a permutation $\sigma$ of $\{1,2, \ldots, n\}$ such that $c\left(y_{i \sigma(j)}\right)= \pm t_{i j}$. Since $\pm y_{i \sigma(j)}$ must begin with its core, it consists only of its core, that is, $y_{i \sigma(j)}= \pm t_{i j}$. Hence $\operatorname{gp}\left(\left\{f_{i j}\right\}_{j=1}^{n}\right)=\operatorname{gp}\left(\left\{y_{i \sigma(j)}\right\}_{j=1}^{n}\right)=Z(A)$. Now since $P K_{0}=K_{0}$, we have that for any $t \in T$ there exist $p, p^{\prime} \in P$ such that $\{t\}=T(p k) \cap T\left(p^{\prime} k\right)$.

$$
f_{t} \in \operatorname{gp}(f T(p k)) \cap \operatorname{gp}\left(f T\left(p^{\prime} k\right)\right)=Z(T(p k)) \cap Z\left(T\left(p^{\prime} k\right)\right)=Z t .
$$

Hence $f_{t}=m t$ for some $m \in Z . m \neq 0$ since $f$ is free. But then it is easy to see that $f k=k$ for all $k \in K_{0}$ implies $m=1, f_{t}=t$. Therefore $f=e$.

Lemma 6. Let $K$ be a proper, non-zero characteristic subgroup of $F$ with basis $B$. Then $\{|b| \mid b \in B\}$ is unbounded.

Proof. We may assume $B$ is Nielsen reduced. Let $Q$ be the minimal Schreier system of coset representatives corresponding to $B$. Suppose $|b|<N$ for all $b \in B$. Suppose $q \in Q$ is such that $|q|>N$. $K$ characteristic implies there exists $0 \neq k \in K$ such that $T(q) \cap T(k)=\varnothing$ and so $q+k$ is a reduced sum not in $Q$. Since $q+k \equiv$ $q \bmod K$ we can write $k=a+\epsilon t+c$, a reduced sum where $q+a \in Q$ and $q+a+$ $\epsilon t \notin Q$. But then for some $q^{\prime} \in Q, b=q+a+\epsilon t-q^{\prime}$ is a $T$-reduced sum, $b \in \pm B$ with $|b|>N$, a contradiction. Therefore $|q| \leqslant N$ for all $q \in Q$. It is easy to see that 
$\left\{\sum_{i=1}^{n} t_{i}\right\}_{n=1}^{\infty}$ is a basis for F. For $n>N . z=\sum_{i=1}^{n} t_{i}-q \in K$ for some $q \in Q . z \neq 0$ since $|q| \leqslant N$. $z$ must contain exactly one occurrence of some $t_{i}$ and hence is primitive in $F$. But then $K=F$. This contradiction completes the proof.

Theorem 4. For $a \in B C, C_{a}:$ Aut $\rightarrow$ Aut is a group monomorphism. $C_{a}$ is epi if and only if $a \in$ Aut.

Proof. If $x \in \operatorname{Ker}\left(C_{a}\right), C_{a}(x)=e \Rightarrow x a=a e=a \Rightarrow x$ is the identity on $K=\underline{\operatorname{gp}}(a)$ and hence on $K_{0}$. Thus $x=e$ by Lemma 5. Hence $C_{a}$ is a monomorphism.

Now suppose $a \in B C$ and $C_{a}$ is onto. $K=\operatorname{gp}(a)$ is characteristic and nonzero. Assume $K \neq F$ and let $B$ be a Nielsen reduced basis for $K$. By Lemma $3, K_{0}$ is contained in $\operatorname{gp}\left(K_{0} \cap B\right)$. Lemma 6 implies $B-\left(K_{0} \cap B\right)$ is infinite so there exists $x \in \operatorname{Aut}(K)$ such that $x$ non trivially permutes the elements of $B-\left(K_{0} \cap B\right)$. But if $x$ were in the image of $C_{a}, x$ would be the restriction to $K$ of an element of Aut $(F)$, say $y$. $y$ would then fix the elements of $K_{0}$ and so be the identity by Lemma 5 . Thus $x$ is not in the image of $C_{a}$ so $C_{a}$ is not onto. Hence $C_{a}$ onto $\Rightarrow K=F \Rightarrow a \in$ Aut.

For the converse, take $a \in$ Aut and note that $x \in A u t \Rightarrow a x=\left(a x a^{-1}\right) a$ so $C_{a}\left(a x a^{-1}\right)=x$.

Corollary 2. For $a \in B V, Y_{a}$ is onto if and only if $a \in$ Aut.

Proof. if $a \in$ Aut then $x \in E \Rightarrow a x=\left(a x a^{-1}\right) a \Rightarrow x=Y_{a}\left(a x a^{-1}\right)$. Hence $a \in$ Aut implies $Y_{a}$ is onto.

Suppose $Y_{a}$ is onto. By Proposition 3 (1) of Section 2, $Y_{a} \mid$ Aut $=C_{a}$ so by Theorem 4 it suffices to show that for $x \in E, Y_{a}(x) \in$ Aut $\Rightarrow x \in$ Aut. Suppose $Y_{a}(x) \in$ Aut. Let $V=\operatorname{gp}(a) . Y_{a}(x)$ free $\left.\Rightarrow x\right|_{V}$ is one to one $\Rightarrow \operatorname{Ker}(x)=0$, since if not $\operatorname{Ker}(x) \cap V \supseteq$ $V(\operatorname{Ker}(x)) \neq 0$ since $\operatorname{Ker}(x) \neq 0 \Rightarrow \operatorname{rank}(\operatorname{Ker}(x))=\omega .\left.\quad x\right|_{v}: V \rightarrow V$ onto implies by Lemma 2, of Section 2 that $x \in$ Aut.

\subsection{Prime Factorization in $\underline{C}$}

In this section we define primes in $\underline{C}$ and $B C$ and prove those results we have on unique prime factorization in $\underline{C}$.

Note that Aut is the group of units of the near ring $E$.

Definition 4. Suppose Aut $\subseteq M \subseteq E$ where $M$ is a multiplicative submonoid of $E$. We say that $p \in M$ is $M$-prime if $p \notin$ Aut and $p=a b, a, b \in M \Rightarrow a$ or $b$ is in Aut. $K$ is prime in $\underline{C}$ if $K=H H^{\prime} \Rightarrow H$ or $H^{\prime}$ is $F$.

Since $B C, B V \supseteq$ Aut, the above definition defines the primes of $B C$ and $B V$. Since $\mathrm{gp}: B C \rightarrow \underline{C}$ and $\mathrm{gp}: B V \rightarrow \underline{V}$ are monoid epimorphisms, the primes of $B C(B V)$ are exactly those elements of $E$ basic for some prime in $C(V)$. Thus $x \in B C(B V)$ is prime if and only if $\underline{\mathrm{gp}}(x)$ is prime in $\underline{C}(V)$.

Theorem 5. (a) Every $k \in B C$ can be written as a finite product of $B C$ primes.

(b) Every $K \in \underline{C}$ can be written as a finite product of primes and any such 
factorization takes the form: $K=\prod_{i=1}^{n} P_{i}$ where $n<|K|, P_{i}$ prime in $\underline{C}$.

Proof. First we show that if $a, b$ are non-units of $B C, \operatorname{gp}(a b)$ is a proper characteristic subgroup of $\mathrm{gp}(a)$. Clearly $\mathrm{gp}(a b)$ is a subgroup of $\mathrm{gp}(a)$. If $\mathrm{gp}(a b)=$ $\underline{\operatorname{gp}(a)}$ then there exists $x \in \bar{E}$ Aut such that $a b x=a \Rightarrow b x=e$ and so $b \in$ Aut. Thus $\overline{\mathrm{gp}}(a b) \subseteq \mathrm{gp}(a)$.

If $x \in \operatorname{Aut}(\mathrm{gp}(a))$ there exists a $y \in$ Aut such that for all $i \geqslant 1 \quad x\left(a_{i}\right)=(a y)_{i}$. Therefore $x(\operatorname{gp}(a b))=\operatorname{gp}\left(\left(\sum x\left(a_{i}\right) s_{i}\right) b\right)=\operatorname{gp}(a y b)=\operatorname{gp}\left(a b C_{b}(y)\right)=\operatorname{gp}(a b)$. Thus $\operatorname{gp}(a b)$ is a proper characteristic subgroup of $\operatorname{gp}(a)$ and so by Lemma $4, \operatorname{Tgp}(a b)|>| \operatorname{gp}(a) \mid$.

From this we have that if $\left\{K_{1}, \ldots, K_{n}\right\}$ are proper characteristic subgroups then $\left|\Pi_{i=1}^{n} K_{i}\right|>n$. Thus if $K \in \underline{C}$ cannot be written as a finite product of primes it can be represented as an arbitrarily long product of proper characteristic subgroups, and so it has arbitrarily large length. This contradiction implies any $0 \neq K \neq F$ can be written as a finite product of primes of the form: $K=\prod_{i=1}^{n} P_{i}$ where $n<|K|$, and $P_{i}$ prime in $\underline{C}$. This proves (b).

Now if $a \in B C$ cannot be written as a finite product of primes, $g p(a)$ can be written as an arbitrarily long product of proper characteristic subgroups. This contradiction proves (a).

Proposition 5. Unique prime factorization holds in $\underline{C}$ if and only if it holds up to multiplication by units in $B C$.

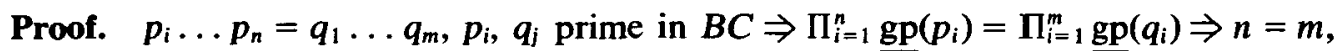
$\operatorname{gp}\left(p_{i}\right)=\operatorname{gp}\left(q_{i}\right)$ for $1 \leqslant i \leqslant n \Rightarrow n=m$, and for all $i, p_{i}=q_{i} x_{i}$ for some $x_{i} \in$ Aut.

If $\Pi_{i=1}^{n} P_{i}=\Pi_{i=1}^{m} Q_{i}$ for $P_{i}, Q_{i}$ prime in $C$, take $p_{i}$ basic for $P_{i}, q_{j}$ basic for $Q_{i}$ for all $i, j$. Then there exists $x \in$ Aut such that

$$
p_{1} \ldots p_{n}=q_{1} \ldots q_{m} x \text {. }
$$

By hypothesis $n=m$ and $p_{i}=q_{i} x_{i} 1 \leqslant i<n, p_{n}=q_{n} x x_{n}$ where $x_{i} \in$ Aut. Hence $n=m$ and $P_{i}=Q_{i}$ for all $1 \leqslant i \leqslant n$.

We now show that a proof of unique prime factorization in $C$ would also give a proof of unique prime factorization in $\underline{V}$. To do this we need a lemma.

Lemma 7. If $K$ is non-zero in $C$ then for any $m \geqslant 1$ there is a basis $B$ of $K$ such that $B \supseteq\left\{b_{1}, \ldots, b_{m}\right\}$ and the $T\left(b_{i}\right)$ are pairwise disjoint.

Proof. Let $B$ be a Nielsen reduced basis for $K$. Take $k_{1}, \ldots, k_{n} \in K_{0}$ such that the $T\left(k_{i}\right)$ are pairwise disjoint. By Lemma 3, if $k_{i} \in \pm B$ then $k_{i}=\epsilon_{i} b_{i 1}+\delta_{i} b_{i 2}$ for $\epsilon_{i}, \delta_{i}= \pm$, and $b_{i j} \in B$. If $b_{i j}=b_{r s}$ for $r \neq i$ then $c\left(b_{i j}\right)$ occurs in the reduced $T$-form of both $k_{i}$ and $k_{r}$, a contradiction. Therefore $\left(B-\left\{b_{i 1} \mid k_{i} \notin \pm B\right\}\right) \cup\left\{k_{i} \mid k_{i} \notin \pm B\right\}=B^{\prime}$ is a basis of $K$ such that $B^{\prime} \cup-B^{\prime} \supseteq\left\{ \pm k_{i}\right\}_{i=1}^{m}$. This proves the lemma.

For $A$, a subset of $F$, define $P_{A} \in E$ by $P_{A}(t)=\left\{\begin{array}{l}0 \text { if } t \in A \\ t \text { if } t \notin A\end{array}\right.$.

We say a subgroup $K \subseteq F$ is projection closed if $P_{A}(K) \subseteq K$ for all $A \subseteq T$. 
Proposition 6. $K \in \underline{C}$ is projection closed if and only if $K \in \underline{V}$.

Proof. Given $K \in \underline{C}$ is projection closed, $w\left(t_{i j}\right)_{j=1}^{n} \in K$ and $\left\{w_{j}\right\}_{j=1}^{n} \subseteq F$. Take $R=\left\{r_{j}\right\}_{j=1}^{n} \subseteq T$ such that $\left\{r_{j}\right\}_{j=1}^{n} \cap\left(\cup_{j=1}^{n} T\left(w_{j}\right)\right)=\emptyset .\left\{r_{j}+w_{j}\right\}_{j=1}^{n}$ is primitive so since $K \in \underline{C}, w\left(r_{j}+w_{j}\right)_{j=1}^{n} \in K$. Since $K$ is projection closed $P_{R}\left(w\left(r_{j}+w_{j}\right)_{j=1}^{n}\right)=w\left(w_{j}\right)_{j=1}^{n} \in$ $K$. Therefore $K \in \underline{V}$.

Note that $K \in \underline{C}$ is projection closed if and only if $P_{R}(K) \subseteq K$ for some finite $R \subseteq T$. If $t \in T$, let $P_{\{t\}}=P_{t}$.

Theorem 6. $K, H \in \underline{C}, K H \in \underline{V}$ implies $K, H \in \underline{V}$. Thus if unique prime factorization holds in $\underline{C}$ it also holds in $\underline{V}$.

Proof. To show $H \in \underline{V}$ take $w\left(t_{i j}\right)_{j=1}^{n} \in H$ and show $P_{t_{i 1}}(w) \in H$. By Lemma 7, we may take $b$ basic for $K$ such that $T\left(b_{i j}\right) \cap T\left(b_{i s}\right)=\emptyset$ for $r \neq s$. $b H=K H$ implies $b w=w\left(b_{i j}\right) \in K H$. Since $K H \in \underline{V}, P_{T\left(b_{i 1}\right)}\left(w\left(b_{i j}\right)\right)=b P_{t_{i 1}}(w)$, which is in $K H$. Therefore $b P_{t_{i 1}}(w)=b h$ for some $h \in H$. $b$ free implies $P_{t_{i 1}}(w)=h \in H$. Thus $H \in \underline{V}$.

Now $E K$ is the verbal subgroup generated by $K$. To show this we need only show $E K$ is a group. Clearly $-(E K) \subseteq E K$. If $f, g \in E$ and $x, y \in K$, then $K \in C$ implies there exists an $f^{\prime} \in E, x^{\prime} \in K$ such that $f x=f^{\prime} x^{\prime}$ and $T\left(x^{\prime}\right) \cap T(y)=\emptyset$. Then clearly there exists a $q \in E$ such that $q\left(x^{\prime}+y\right)=q x^{\prime}+q y=f^{\prime} x^{\prime}+g y=f x+g y$. Thus $E K \in \underline{V}$.

Let $E K=\bar{K} \in \underline{V}$ and let $\bar{k}, k, h$ be basic for $\bar{K}, K, H$ respectively.

$$
E \cdot k \cdot E=E \cdot\left(K^{\omega}\right)=\bar{K}^{\omega}=\bar{k} \cdot E
$$

The middle equality comes from the fact that $E K=\bar{K}$ and for any $x \in \bar{K}^{\omega}, x=$ $f\left(\Sigma k_{i} s_{i}\right)$ where $k_{i} \in K$ and the $T\left(k_{i}\right)$ are pairwise disjoint. Hence

$$
\bar{K} H=\underline{\mathrm{gp}}(\bar{k} h E)=\underline{\mathrm{gp}}(\bar{k} E h E)=\underline{\mathrm{gp}}(E k E h E)=\underline{\mathrm{gp}}(E k h E)=\underline{\mathrm{gp}}(k h E)=K H .
$$

Therefore $\bar{K} H=K H$ and $\bar{K} \supseteq K$. By Theorem $3, \bar{K}=K$ so $K \in \underline{V}$. This proves our first statement.

Now from the above it is easy to see that $P$ prime in $\underline{V}$ implies $P$ prime in $\underline{C}$. Thus if unique prime factorization holds in $\underline{C}$ it also holds in $\underline{V}$.

In the remainder of this section we introduce a notion due to Frohlich (see (1)). This, it happens, is important in proving unique prime factorization in $\underline{V}$ and might well prove important in constructing a proof of unique prime factorization in $\underline{C}$.

Definition 5. For $K, K^{\prime} \in \underline{C}$, define $K \backslash K^{\prime}=\sup \left\{H \in C \mid K H \subseteq K^{\prime}\right\}$.

Clearly $K \backslash K^{\prime} \in \underline{C}$. Let $k$ be basic for $K$. For $H \in \bar{C}, K H=k H$. If $x \in K \backslash K^{\prime}$, $x=\sum_{i=1}^{n} h_{i}$ where $h_{i} \in H_{i}$, and $K H_{i} \subseteq K^{\prime}$. Thus $k x=\sum_{i=1}^{n} k h_{i} \in K^{\prime}$, and so

$$
K\left(K \backslash K^{\prime}\right) \subseteq K^{\prime}
$$

$K \backslash K^{\prime}$ is therefore the unique maximal characteristic subgroup $H$ such that $K H \subseteq K^{\prime}$.

If we replace $\underline{C}$ by $\underline{V}$ in the above definition we get a slicing operation on $\underline{V}$. (This is actually the restriction to $\underline{V}$ of the slicing operation on $\underline{V}$ though there is not space here to prove this. For a proof, see (9), Chapter V, Section 3.) The essential step in proving unique prime factorization in $\underline{V}$ is Lemma 23.21 of Varieties of Groups, which 
in our notation becomes:

$$
V, V^{\prime} \in \underline{V} \text { and } U \underline{\underline{Z}} V \Rightarrow U \backslash\left(V V^{\prime}\right)=(U \backslash V) V^{\prime}
$$

It happens that the same lemma extended to $\underline{C}$ will yield unique prime factorization in C.

Theorem 6. Suppose that if $H, K, K^{\prime} \in \underline{C}$ and $H \underline{q} K$ then $H \backslash\left(K K^{\prime}\right)=(H \backslash K) K^{\prime}$. Then unique prime factorization holds in $\underline{C}$.

Proof. First we show that under our hypotheses, $K, K^{\prime}, H \in \underline{C}$ implies that $K H=K^{\prime} H \Rightarrow K=K^{\prime}$. Assume $K H=K^{\prime} H$ and $K \underline{\varnothing} K^{\prime}$. Since $H \subseteq K \backslash\left(K^{\prime} H\right)$, $K\left(K \backslash\left(K^{\prime} H\right)\right)=K H=K^{\prime} H$. By hypothesis, since $K \underline{\varnothing} K^{\prime}, K\left(K \backslash\left(K^{\prime} H\right)\right)=K\left(K \backslash K^{\prime}\right) H=$ $K H$. By the easy cancellation law, $\left(K \backslash K^{\prime}\right) H=H=F H$. Hence by Theorem $3, K \backslash K^{\prime}=$ $F$. But $K\left(K \backslash K^{\prime}\right) \subseteq K^{\prime}$ implies $K \subseteq K^{\prime}$, a contradiction. Thus if $K H=K^{\prime} H$, we must assume $K \subseteq K^{\prime}$ or $K^{\prime} \subseteq K$. By Theorem 3 again, $K=K^{\prime}$, and we have our right cancellation law.

Now suppose $P H=Q K$, where $P, Q, H, K \in \underline{C}, P, Q$ primes. Suppose $P \underline{q} Q$. Then $P \backslash(Q K)=(P \backslash Q) K . H \subseteq P \backslash(Q K) \Rightarrow P(P \backslash(Q K))=P(P \backslash Q) K=Q K$ so by the right cancellation law, $P(P \backslash Q)=Q$. $P, Q$ are prime implies $P \backslash Q=F$ so $P \subseteq Q$, a contradiction. Hence $P \subseteq Q$, and by symmetry, $P \supseteq Q$. Hence $P=Q$, proving the theorem.

\subsection{Cancellation in $B C$}

Although we do not have a proof that both cancellation laws hold in $\underline{C}$, as an indication that this is true we can prove that both cancellation laws hold in $B C$. This result reduces the problem of proving the right cancellation law in $C$ to the problem of showing that if $a, a^{\prime}, b \in B C$ and $a b=a^{\prime} b x$ for some $x \in A u t$, then $x=C_{b}(y)$ for some $y \in$ Aut.

Definition 6. $x \in F$ is indecomposable if and only if $x=n y, n \geqslant 1 \Rightarrow n=1$.

The following results are well known:

1. For any $z \in F$, there exists a unique indecomposable $x \in F$ such that $z=n x$ for some $n \geqslant 1$. We denote this $x$ by $I(z)$.

2. Any indecomposable $x=g+y-g$, where this is a reduced sum for some $g \in F$ and some cyclically reduced indecomposable, $y$.

3. If $y$ is a cyclically reduced indecomposable then all $y$ 's cyclic permutations are distinct.

4. If $x, y$ are indecomposable and $x \neq \pm y$, then $\{x, y\}$ is free. Proof: For clearly $\operatorname{gp}(\{x, y\})$ is non-cyclic. Since free groups of finite rank are Hopfian, $\{x, y\}$ is a basis for $\operatorname{gp}(\{x, y\})$.

Lemma 8. Suppose $x, y \in F$ are cyclically reduced indecomposables and $|x| \geqslant|y|$. Then if for $k \geqslant 1$ arbitrarily large we have a diagram of the form:

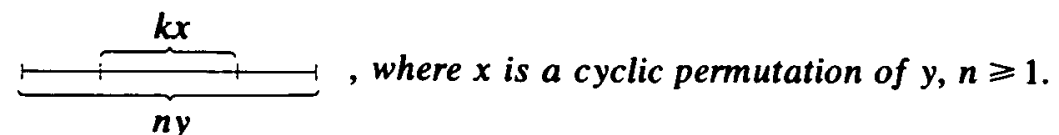


Proof. Take a $k \geqslant 8$. We have a diagram of form: $\underbrace{\overbrace{n}^{4 x} 4 x}_{n y}$ where $n \geqslant 1$.

Therefore $4 x=a+k_{1} y+b=c+k_{2} y+d$ where both sums are reduced; $k_{1}, k_{2} \geqslant 2 ; b, d$ are proper initial segments of $y ; a, c$ are proper terminal segments of $y$, and $y=b+c$, a reduced sum.

If $a \neq c$ then it is easy to see we have a diagram: $\underbrace{\underbrace{c_{k} t_{1}}_{y}}_{y}$, where $k, h \neq 0$.

Therefore $y=k+h=h+k$, so $y$ is a proper cyclic permutation of itself and hence not indecomposable, a contradiction. Thus $a=c$ so $b=d, k_{1}=k_{2}$ and $4 x=$ $\left(k_{1}+1\right)(a+b) . a+b$ is a cyclic permutation of $y$ and hence indecomposable. Thus $4=k_{1}+1, a+b=x$.

Lemma 9. Suppose $u, w$ are non-zero in $F$ and $I(u) \neq \pm I(w)$. Let $w=g+w^{\prime}-g, a$ reduced sum where $w^{\prime}$ is cyclically reduced. Then for any $\epsilon, \delta= \pm$ there is an $N \geqslant 1$ such that for $k, k^{\prime} \geqslant N$ the reduced $T$-form of $k(\epsilon w)+u+k^{\prime}(\delta w)$ has $g+\epsilon w^{\prime}$ for an initial segment and $\delta w^{\prime}-g$ for a terminal segment.

Proof. For $N \geqslant 1$ let $x_{N}=N e w+u+N \delta w$. By Remark 4, $\{w, u\}$ is free so (1) $\left\{\left|x_{N}\right| \mid N \geqslant 1\right\}$ is unbounded. Suppose $x_{N}$ never has both $g+\epsilon W^{\prime}$ for an initial segment and $\delta w^{\prime}-g$ for a terminal segment. Then we always have $\left|x_{N}\right| \leqslant$ $4|g|+|u|+2 N\left|w^{\prime}\right|-2(N-1)\left|w^{\prime}\right|=4|g|+|u|+2\left|w^{\prime}\right|$, contradicting (1). This proves the lemma.

Along the same lines as Lemma 9 we have:

Remark 5. Let $u, w, \epsilon, \delta$ be as in Lemma 9. Then there exists an $N \geqslant 1$ such that for $M \geqslant N, M \epsilon w+u$ has initial segment $g+\epsilon w^{\prime}$ and $u+M \delta w$ has terminal segment $\delta w^{\prime}-g$.

Theorem 7. Suppose $a, a^{\prime} \in E$ are free and $b \in B C$. Then if $a b=a^{\prime} b$, we have $a=a^{\prime}$.

Proof. $a b=a^{\prime} b$ implies $a w=a w^{\prime}$ for all $w \in \underline{g p}(b)=B \in \underline{C}$. Pick $w \in B$ such that $t_{i} \notin T(w)$ and $w=t_{j}+w^{\prime}$, a $T$-reduced sum for some $t_{j} \in T$. For $k \geqslant 1$, define $x_{k} \in$ Aut such that $x_{k}(t)=\left\{\begin{array}{l}t \text { if } t \neq t_{j} \\ k t_{i}+t_{j} \text { if } t=t_{j}\end{array}\right.$.

Let $w_{k}=x_{k} w$. Then for $k \geqslant 1 w_{k}=\sum_{r=1}^{H}\left(k\left(\epsilon_{r} t_{i}\right)+v_{r}\right)$ is a $T$-reduced sum where $\epsilon_{1}=+, 0 \neq v_{r} \in \operatorname{gp}(T(w))$ for $1 \leqslant r<H$, and $H$ is the number of occurrences of $\pm t_{j}$ in w. $v_{H} \in \operatorname{gp}(T(w))$ may be 0 .

We claim that: $u \in \mathrm{gp}(a T(w))$ implies $I(u) \neq \pm I\left(a_{i}\right)$ and $u \in \mathrm{gp}\left(a^{\prime} T(w)\right)$ implies $I(u) \neq \pm I\left(a_{i}^{\prime}\right)$.

To prove the first implication assume $I(u)= \pm I\left(a_{i}\right)$. Then $n a_{i} \in \operatorname{gp}(a T(w))$ for some $n \geqslant 1$, contradicting the freeness of $\left\{a_{i}\right\} \cup a T(w)$. The second statement of our claim is proved similarly. 
Now $a_{i}=g+M x-g$ and $a_{i}^{\prime}=h+M^{\prime} y-h$, where these are both reduced sums, $M, M^{\prime} \geqslant 1$, and $x, y$ are cyclically reduced indecomposables. By our claim, for all $1 \leqslant r \leqslant H, I\left(a v_{r}\right) \neq \pm I\left(a_{i}\right)$ and $I\left(a^{\prime} v_{r}\right) \neq \pm I\left(a_{i}\right)$.

By repeatedly using Lemma 9 and Remark 5 on both sides of the equation $a w_{k}=a^{\prime} w_{k}$ we have if $|x| \geqslant|y|$, a diagram of form

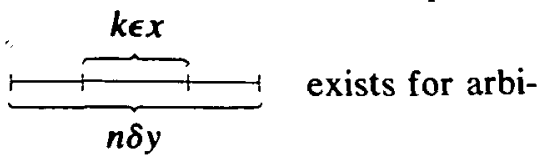

trarily large $k$. If $|x| \leqslant|y|$ we get the same situation with $x$ and $y$ interchanged. Therefore Lemma 8 applies showing that $x$ is a cyclic permutation of $\pm y$. Hence $|x|=|y|$.

For $k$ sufficiently large, $k M|x|+c=\left|a w_{k}\right|=\left|a^{\prime} w_{k}\right|=k M^{\prime}|y|+d$, where $c$ and $d$ are constants not depending on $k$. Using the fact that $|x|=|y|$, and letting $k$ go to infinity, we get $M=M^{\prime}$.

Now suppose $|g| \neq|h|$. Then $|g|<|h|$ or $|g|>|h|$. Suppose $|g|>|h|$. Since $w_{k}$ begins with $k t_{i}$, for $k$ sufficiently large, we get from the equation, $a w_{k}=a^{\prime} w_{k}$, a diagram of the form

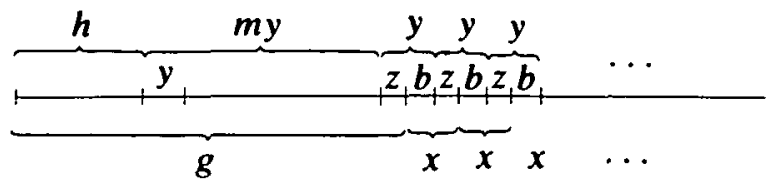

where $\boldsymbol{m} \geqslant 0$ and $z$ may be zero.

If $z \neq 0, y=z+b, x=b+z$, reduced sums and $g+x-g$ is a reduced sum. Thus $(h+m y+z)+(b+z)+(-z-m y-h)$ is a reduced sum, which it isn't. Hence $z=0$ and $x=y$. But this means that since $g+x-g$ is a reduced sum we must have $m=0$ and $h=g$. Therefore

$$
a_{i}=-g+M x-g=h+M^{\prime} y-h=a_{i}^{\prime} .
$$

Since $t_{i}$ was arbitrary, $a=a^{\prime}$.

Corollary 3. Both cancellation laws hold in $B C$.

\section{REFERENCES}

(1) A. FroHLICH, Distributively generated near rings (I): Ideal Theory, Proc. London Math. Soc. (3) 8 (1958), 74-94.

(2) A. Frohlich, Distributively generated near rings (II): Representation Theory, Proc. London Math. Soc. (3) 8 (1958), 95-108.

(3) R. C. LYNDON and P. E. SCHUPP, Combinatorial Group Theory (Springer-Verlag, 1977).

(4) W. Magnus, A. Karrass and D. Solitar, Combinatorial Group Theory (Interscience tracts in Pure and Applied Mathematics, Vol. XIII, Interscience Publishers, N:Y., 1966).

(5) H. NEUMANN, On varieties of groups and their associated near rings, Math. Z. 65 (1956), 36-69.

(6) H. NEUMANN, Varieties of Groups (Springer-Verlag, 1967). 
(7) B. H. NeumanN, H. NeumanN and P. M. NeumanN, Wreath products and varieties of groups, Math. Z. 80 (1962), 44-62.

(8) V. ThaRMARATNAM, Endomorphism near rings of relatively free groups, Math. Z. 113.

(9) R. W. ZeAmER, On Near Rings Associated with Free Groups, (Ph.D. thesis, McGill University, Montreal, Canada, 1977).

Queen Mary College 